\title{
Field-Effect Phototransistors Based on Graphene-Quantum Dot Hybrids
}

\author{
Heng Zhang ${ }^{1}$, Ya Ting Zhang ${ }^{1, a}$ \\ ${ }^{1}$ Institute of Laser \& Opto-Electronics, College of Precision Instruments and Opto-electronics Engineering, Tianjin \\ University, Tianjin 300072, people's Republic of China
}

\begin{abstract}
Field effect photo-transistors (FEpTs) based on graphene-PbSe quantum dot (QD) hybrids have been designed and fabricated. By electrical and photoelectrical measurements, the carrier mobilities reached to $1621 \mathrm{~cm}^{2} \mathrm{~V}^{-}$ ${ }^{1} \mathrm{~s}^{-1}$ for electrons and $1228 \mathrm{~cm}^{2} \mathrm{~V}^{-1} \mathrm{~s}^{-1}$ for holes, the photoresponsivity (R) achieved to $1 \mathrm{AW}^{-1}$, and the photoresponse time $\left(\tau_{1}\right)$ was $0.7 \mathrm{~s}$ when the photocurrent came to about $80 \%$. Therefore, the FEpTs based on graphene-QD hybrids have shown broad application prospects.
\end{abstract}

\section{Introduction}

Field effect phototransistors based on graphenesemiconductor quantum dot (QD) hybrids have achieved high performances, and have attracted great attention for a number of near infrared (NIR) detector applications because of their low cost and low energy consumption, increased flexibility, and easy fabrication and easy integration [1]. The photoconductive gain of FEpTs based on graphene-QD hybrids has reached up to $10^{8}$ and was several orders of magnitude larger that of any graphene FETs or QD FETs reported before [2-5]. The excellent performance of extra high mobility and responsivity of FEpTs based on graphene-quantum dots is mainly attributed to the large absorbance of PbSe QDs and the fast transport in graphene [6]. The intrinsic properties of these two materials provide an opportunity for FEpTs to achieve ultrahigh performances. Recently, some architecture of FEpTs have been designed and fabricated based on graphene-QD hybrids, and the electrical and optical characteristics have been studied. The results exhibited high mobilities, high photoconductive gain and high responsivities. Here, we designed and fabricated the structure of FEpTs based on graphene-PbSe QD hybrids. With the experiment and theory calculation, the carrier mobilies were measured to be $1621 \mathrm{~cm}^{2} \mathrm{~V}^{-1} \mathrm{~s}^{-1}$ for electrons and $1228 \mathrm{~cm}^{2} \mathrm{~V}^{-1} \mathrm{~s}^{-1}$ for holes, the photoresponsivity $\mathrm{R} \sim 1 \mathrm{AW}^{-1}$, and the photoresponse time $0.7 \mathrm{~s}$ when the photocurrent reached to almost $80 \%$. Therefore, the FEpTs based on graphene-QD hybrids will have broad application prospects.

\section{Experiments}

\subsection{Structural design}

\footnotetext{
${ }^{\text {a }}$ Ya-Ting Zhang: yating@tju.edu.cn
}

Graphene provides a channel that can transport carriers effectively (high mobility) yet with weak capacity of photo absorption. On the other hand, QDs serve as providers of photo-induced carriers with low mobility. Thus the device designed with QDs as light absorption layer and graphene as charge transfer layer can achieve ultrahigh photoresponsivity.

PbSe QDs were synthesized using a wet chemical method, and a typical synthesizing process was detailed in reference [7].The absorption and photoluminescence (PL) spectrum of QDs is shown in figure 1(a), according to which the absorption and PL peaks were $1616 \mathrm{~nm}$ and $1645 \mathrm{~nm}$ correspondingly. And the architecture is given by figure $1(\mathrm{~b})$.
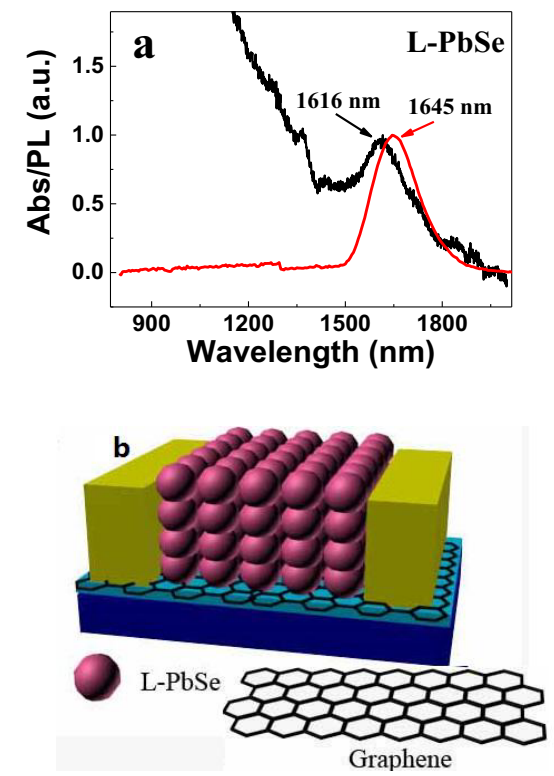

Figure 1.(a) The absorption and PL spectra of PbSe QDs. (b) Schematic diagram 


\subsection{Device fabrication and measurements}

\subsubsection{Electrodes}

The contact between metallic electrode and graphene not only changes the doping of graphene but also affects its electronic structure and Fermi energy. Therefore the contact have an impact on graphene's electrical characteristics near the electrode, eventually altering the property of devices. Researches have shown that $\mathrm{Au}$ is the only one whose forbidden band between graphene is 0 among several choices such as $\mathrm{Cu}, \mathrm{Ag}, \mathrm{Al}$ and so on [8]. Thus Au was utilized as the electrode material to reduce the influence to the device.

\subsubsection{Fabrication of devices}

FEpTs were fabricated on heavily doped, n-type $\mathrm{Si}$ substrate with a $300 \mathrm{~nm}$ thick $\mathrm{SiO}_{2}$ capping layer on top and a predefined back gate electrode at the bottom. The graphene sheet was grown by chemical vapor deposition (CVD) and was transferred to the substrate [9]. Then, Au source and drain electrodes with a thickness of $500 \mathrm{~nm}$ were thermally evaporated using a shadow mask. Subsequently, four layers of PbSe QDs were deposited by using a layer-by-layer method. Each layer was prepared as follows: one drop of PbSe QD solution $\left(30 \mathrm{mg} \mathrm{ml}^{-1}\right)$ was deposited on a spinning substrate at a speed of 2000 rpm for 60 s and left for $20 \mathrm{~s}$ to dry. Three drops of $2 \%$ (vol.) ethanedithiol (EDT) solution were then deposited on the rotating substrate at the same spin speed for ligand exchange, followed by two drops of acetonitrile and two drops of toluene.

\subsubsection{Measurements}

In the electrical measurements, a bias voltage $\left(V_{S D}\right)$ was applied over the source (ground connection) and drain electrodes using a Keithley 2400. The channel current flowing into the drain is $I_{D}$, which was also measured by the Keithley 2400. An applied gate voltage $\left(V_{G}\right)$ electrically connected the gate electrode to the source using HP6030A. During the photo electrical measurement, an $808 \mathrm{~nm}$ continuous-wave $(\mathrm{cw})$ laser was used to provide incident light.

\section{Results and discussion}

\subsection{Transfer characteristics}

To illustrate the role of graphene, a comparison measurement in conductivity was performed on the FEpTs with a single layer of graphene and that without it. Figure 2 shows the increments in channel current as the bias voltage increases gradually for 300s. Figure 2 (a) displays the FEpTs conductivity without a layer of graphene and the $I_{D}$ rose from $4.24 \times 10^{-4} \mu A$ to $0.0123 \mu \mathrm{A}$, the increments is several $\mathrm{nA}$ while the $V_{S D}$ increased from $5 \mathrm{~V}$ to $150 \mathrm{~V}$. Figure (b) shows the FEpTs conductivity with a graphene layer, the $V_{S D}$ increased from $5 \mathrm{~V}$ to $25 \mathrm{~V}$, while the $I_{D}$ increased from $5.97 \mathrm{~mA}$ to
$35.54 \mathrm{~mA}$, the increments have reached to several $\mathrm{mA}$, which is five orders higher than the former FEpTs. The results demonstrate that the graphene have a great contribution on conductivity of FEpTs.
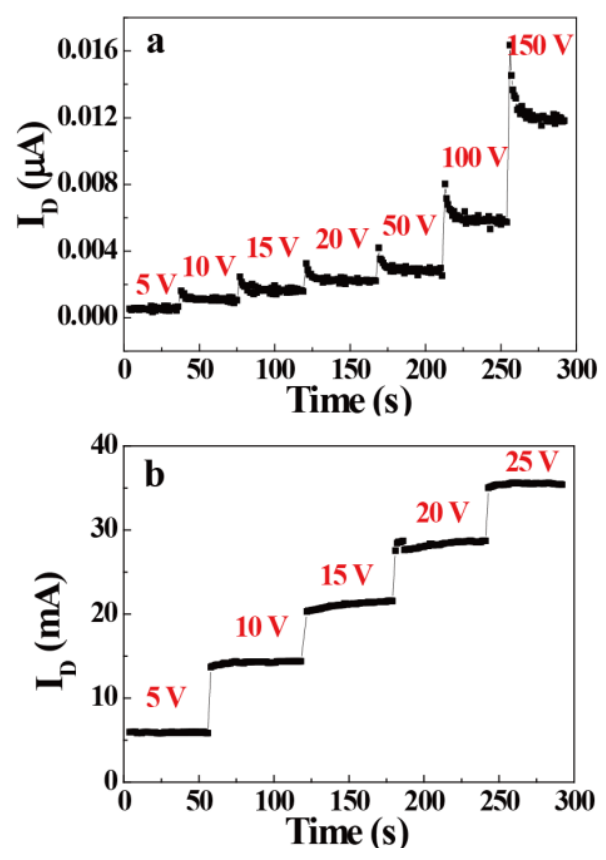

Figure 2. Conductivity respone to bias voltages, (a) QDs FEpTs without graphene, (b) QDs FEpTs with a layer of graphene.

\subsection{Electrical properties of the FEpTs}

Electrical measurements were performed at ambient conditions at room temperature.
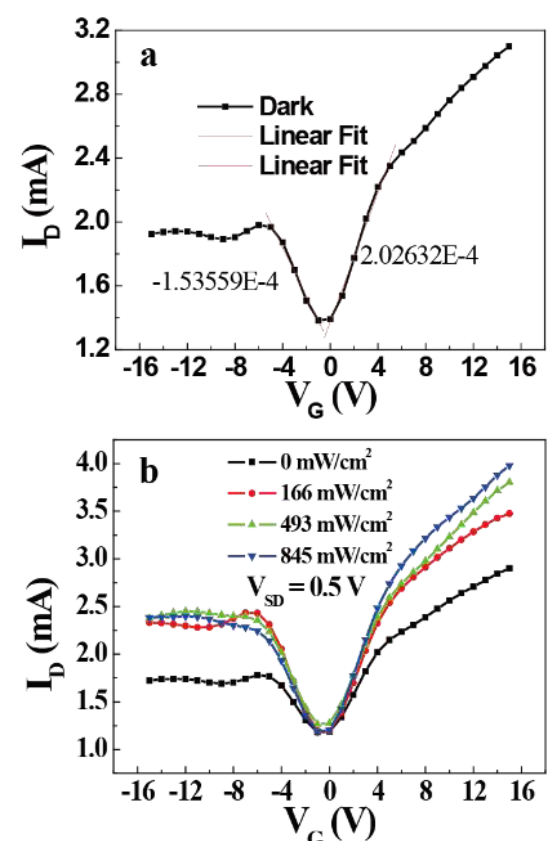

Figure 3. (a) Transfer characteristics $\left(I_{D}\right.$ versus $V_{G}$ ) in the dark with a constant $V_{S D}=0.5 \mathrm{~V}$ and fitting curves. (b) transfer characteristics $\left(I_{D}\right.$ versus $\left.V_{G}\right)$ with a constant $V_{S D}=0.5 \mathrm{~V}$ with illumination of $0 \mathrm{mWcm}^{-2}, 166 \mathrm{mWcm}^{-2}, 493 \mathrm{mWcm}^{-2}$, $845 \mathrm{mWcm}^{-2}$. 
Figure 3 exhibits the transfer characteristics of FEpTs at the bias voltage of $0.5 \mathrm{~V}$. The bipolarity is observed.

Based on FET theory, when $V_{G}>>V_{S D}$, the transfer characteristics is in the linear region because of the constant hole mobility (in the third quadrant) and electron mobility (in the first quadrant) as shown in figure 3 (a). The minimum point in a transfer curve corresponds to the Dirac point of graphene [6]. After modified by QDs, the minimum point slightly shifted. We attributed the slight shift to the internal barrier formed by the capping ligands, which prevented carriers from transferring from the QDs to graphene.

The relation between the current change of $\Delta I_{D}$ and the light-induced gate voltage $\Delta V_{G}$ [9] can be shown as:

$$
\Delta I_{D}=\frac{W \mu C_{O X}}{L} \cdot V_{S D} \cdot \Delta V_{G}
$$

Where $\mathrm{W}$ and $\mathrm{L}$ are the width and length of the channel, respectively and $C_{O X}$ is the capacitance of the gate dielectric per unit area. $\mu$ is the mobility of the carrier. The $\mu$ can be deduced from the equation (1):

$$
\mu=\frac{L}{W C_{O X} V_{S D}} \cdot \frac{\partial I_{D}}{\partial V_{G}}
$$

The slope of $\partial I_{D} / \partial V_{G}$ can be obtained by linearly fitting the transfer characteristics at low $V_{S D}$ and the mobility can be calculated according to equation (2). For $\mathrm{W}=2.5 \mathrm{~mm}, \mathrm{~L}=0.1 \mathrm{~mm}, V_{S D}=0.5 \mathrm{~V}, \mathrm{C}_{\mathrm{ox}} \sim 100 \mathrm{pFcm}^{-2}$, the hole mobility and electron mobility were calculated to be $1228.0 \mathrm{~cm}^{2} \mathrm{~V}^{-1} \mathrm{~s}^{-1}$ and $1621.0 \mathrm{~cm}^{2} \mathrm{~V}^{-1} \mathrm{~s}^{-1}$.

Figure 3 (b) shows the transfer characteristics of FEpTs at the bias voltage of $0.5 \mathrm{~V}$ with the irradiance of 0 $\mathrm{mWcm}^{-2}, 166 \mathrm{mWcm}^{-2}, 493 \mathrm{mWcm}^{-2}, 845 \mathrm{mWcm}^{-2}$. The profile of the transfer curves kept to the same with and without the illumination of laser. When the device was under illumination, QDs absorbed the light to generate the photo-induced carriers. And when the illumination increased, more photo-induced carriers were generated and separated into holes and electrons in the electric field.

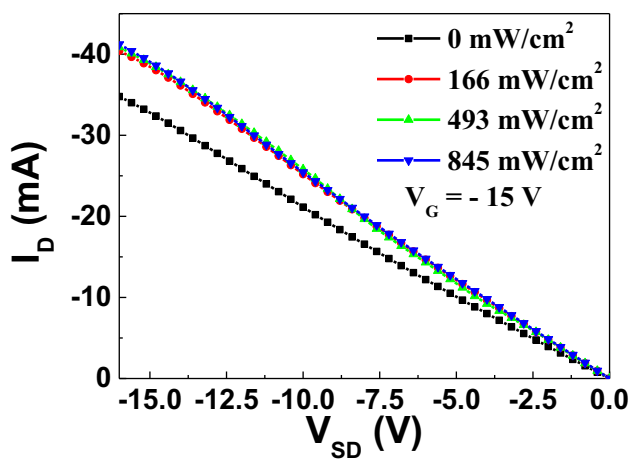

Figure 4. Output characteristics $\left(I_{D}\right.$ versus $\left.V_{S D}\right)$ at the gate voltage of $-15 \mathrm{~V}$ with illuminations of $0 \mathrm{mWcm}^{-2}, 166$ $\mathrm{mWcm}^{-2}, 493 \mathrm{mWcm}^{-2}, 845 \mathrm{mWcm}^{-2}$.

Figure 4 shows the output characteristics of FEpTs, at gate voltage of $-15 \mathrm{~V}$ in the third quadrant, with irradiance of $0 \mathrm{mWcm}^{-2}, 166 \mathrm{mWcm}^{-2}, 493 \mathrm{mWcm}^{-2}, 845$ $\mathrm{mWcm}^{-2}$. The channel current went up with the bias voltage increasing, as the field generated by bias voltage drives the electrons and holes in the graphene to flow, forming the current. And the current was increasing with the rise of irradiance. Little increase of channel current, when the irradiance rose from $166 \mathrm{mWcm}^{-2}$ to 845 $\mathrm{mWcm}^{-2}$, is attributed to a saturation region the channel current reaches.

The physical mechanism of light sensing is described as follows. Under illumination, photo-induced excitons were generated in $\mathrm{PbSe}$ QDs and then separated into free electrons and holes. Because of the driving effect of the gate voltage, holes (or electrons depending on gate voltage) transferred from the QDs to graphene where carriers were transported effectively. Equal numbers of electrons (or holes) remaining in the QDs formed an electric field in the direction opposite to the gate electric field. This additional photo-induced gate voltage is termed the 'light gating effect'. Because of this effect, horizontal shifts in the $\Delta V_{G}$ can be used for FEpTs to calibrate the irradiance $\left(\mathrm{E}_{e}\right)[10]$ :

$$
\Delta V_{G}=\alpha E_{e}^{\beta}
$$

Where $\alpha$ and $\beta$ are constants which reflected the situation of carriers transporting from QDs to graphene. The responsivity $(\mathrm{R})$ of a FEpT is defined using $\Delta I_{D}$ and is

$$
R=\frac{I_{\text {ill }}-I_{\text {Dark }}}{P}=\frac{\Delta I_{D}}{P}
$$

Where $I_{i l l}$ and $I_{\text {Dark }}$ are the channel current values under light and dark, respectively, $\mathrm{P}=\mathrm{AE}_{e}$ is the incident optical power, and A is the illumination area. Substituting equation (2) into (4) gives

$$
\begin{gathered}
R=\frac{\alpha C_{o x} V_{s d} \mu}{L^{2}} E_{e}^{\beta-1} \\
\log (R)=\log \left(\frac{\alpha C_{o x} V_{s d} \mu}{L^{2}}\right)+(\beta-1) \log \left(E_{e}\right)
\end{gathered}
$$

According to the calculation of theory, the $\mathrm{R}$ is in the order scale of $1 \mathrm{AW}^{-1}$.

\subsection{Photoresponse time}

Though the responsivity of graphene-QDs based FEpTs has been measured experimentally, the photoresponse time is an important parameter as well.

The transient photocurrent response of the FEpTs to on/off illumination was measured as shown in figure 5 (a) where the irradiance was $845 \mathrm{mWcm}^{-2}$, at the wavelength of $808 \mathrm{~nm}$, under $V_{S D}=0.01 \mathrm{~V}, V_{G}=3 \mathrm{~V}$, and the on/off time were both $24 \mathrm{~s}$.

The channel current increased sharply once the light was switched on, then the increase slowed down during the illuminating time. On the contrary, when the light was switched off, the current dropped exponentially to the initial state. 

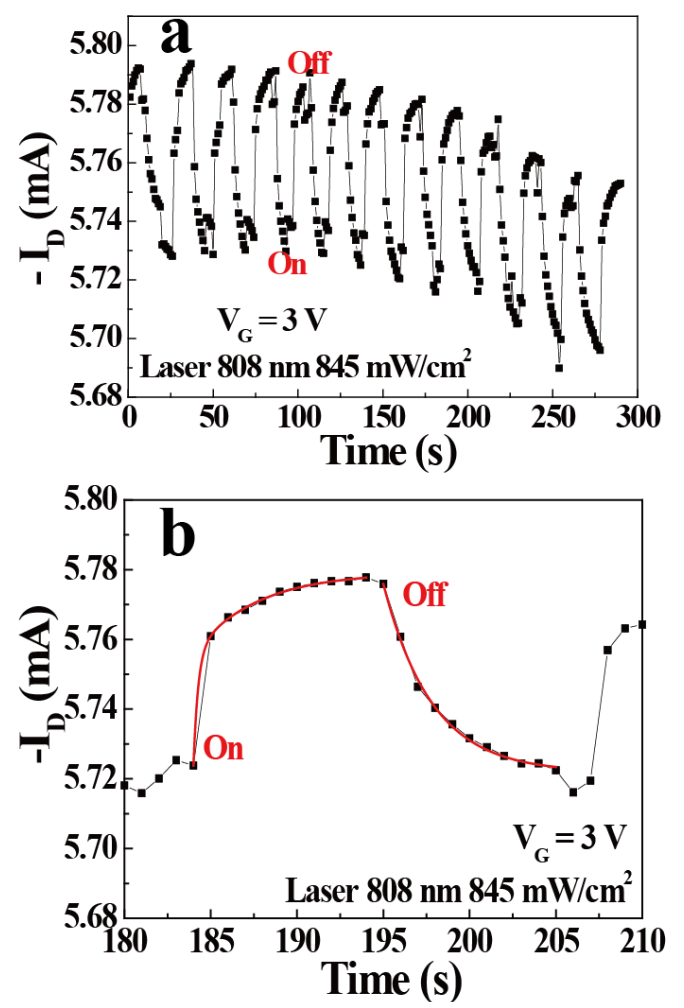

Figure 5. (a) The photocurrent of FEpTs to on/off illumination for various cycles where $V_{S D}=0.01 \mathrm{~V}$ and $V_{G}=3 \mathrm{~V}$. The irradiance was $845 \mathrm{mWcm}^{-2}$, the wavelength was $808 \mathrm{~nm}$ and the on/off time was $24 \mathrm{~s}$. (b) The fitting result of a cycle.

The process of on/off illumination could be fitted using the exponential equation:

$$
I_{D}=\left\{\begin{array}{l}
\Delta I_{1} \exp \left(-\frac{t}{\tau_{1}}\right)+\Delta I_{2} \exp \left(-\frac{t}{\tau_{2}}\right)+I_{\text {Dark }} \\
\Delta I_{1}\left[1-\exp \left(-\frac{t}{\tau_{3}}\right)\right]+I_{\text {Dark }}
\end{array}\right.
$$

Where the physical meaning of $\tau$ refers to the time of carriers transferring between the QDs and graphene sheets, that is, relaxation time. Figure 5 (b) shows the fitting result of a cycle according to the exponential function. The time constants $\tau_{1}$ and $\tau_{2}$ were calculated to be $0.7 \mathrm{~s}$ and $2.4 \mathrm{~s}$ respectively. As the photocurrent has taken the proportion of almost $80 \%$ during the time of $\tau_{1}=0.7 \mathrm{~s}$, the photoresponse property of the FEpTs based on graphene and QDs is remarkable.

\section{Conclusions and summary}

We combined the advantages of graphene with high carrier mobility and quantum dots with large absorbance at the same time, designed the structure of graphenebased FEpTs with four layers of PbSe QDs. Then its electrical and optical properties were studied, the output and transfer curves were plotted, and the photoresponse characteristics were analyzed. Furthermore, we calculated the mobility, photoresponsivity and relaxation time: $\mu_{e}=1621.0 \mathrm{~cm}^{2} \mathrm{~V}^{-1} \mathrm{~s}^{-1}, \quad \mu_{h}=1228.0 \mathrm{~cm}^{2} \mathrm{~V}^{-1} \mathrm{~s}^{-1}$, $\mathrm{R} \sim 1 \mathrm{AW}^{-1}, \tau_{1}=0.7 \mathrm{~s}, \tau_{2}=2.4 \mathrm{~s}$. Through the data, it can be concluded that FEpTs based on the graphene and PbSe QDs achieved excellent performances with high mobility of electrons and holes and great light responsivity. It is ensured that the FEpTs based on graphene-quantum dot hybrids will have a promising future.

\section{References}

1. X. Song, Y. Zhang, R. Wang, M. Cao, Y. Che, J. Wang, H. Wang, L. Jin, H. Dai, X. Ding, G. Zhang, J. Yao, Bulk- and layer-heterojunction phototransistors based on poly 2-methoxy-5-(2 '-ethylhexyloxy-pphenylenevinylene) and $\mathrm{PbS}$ quantum dot hybrids. Applied Physics Letters 106, (2015); published online EpubJun 22 (10.1063/1.4922917).

2. S. Yigen, V. Tayari, J. O. Island, J. M. Porter, A. R. Champagne, Electronic thermal conductivity measurements in intrinsic graphene. Physical Review $B$ 87, (2013); published online EpubJun 27

3. C. P. Church, E. Muthuswamy, G. M. Zhai, S. M. Kauzlarich, S. A. Carter, Quantum dot Ge/TiO2 heterojunction photoconductor fabrication and performance. Applied Physics Letters 103, (2013); published online EpubNov 25

4. D. Reddy, L. F. Register, G. D. Carpenter, S. K. Banerjee, Graphene field-effect transistors. J Phys D Appl Phys 44, (2011); published online EpubAug 10

5. G. Konstantatos, I. Howard, A. Fischer, S. Hoogland, J. Clifford, E. Klem, L. Levina, E. H. Sargent, Ultrasensitive solution-cast quantum dot photodetectors. Nature 442, 180-183 (2006); published online EpubJul 13

6. Y. Zhang, X. Song, R. Wang, M. Cao, H. Wang, Y. Che, X. Ding, J. Yao, Comparison of photoresponse of transistors based on graphene-quantum dot hybrids with layered and bulk heterojunctions. Nanotechnology 26, (2015); published online EpubAug 21 (10.1088/0957-4484/26/33/335201).

7. Y. Zhang, M. Cao, X. Song, J. Wang, Y. Che, H. Dai, X. Ding, G. Zhang, J. Yao, Multiheterojunction Phototransistors Based on Graphene-PbSe Quantum Dot Hybrids. The Journal of Physical Chemistry C 119, 21739-21743 (2015)10.1021/acs.jpcc.5b07318).

8. A. Varykhalov, M. R. Scholz, T. K. Kim, O. Rader, Effect of noble-metal contacts on doping and band gap of graphene. Physical Review B 82, (2010); published online EpubSep 1

9. R. Wang, Y. T. Zhang, J. Q. Yao, Abnormal Temperature Dependence of Mobility in a Disordered System With Traps: Experiment and Theory. IEEE Photonics Journal 7, 1-8 (2015)10.1109/jphot.2015.2423561).

10. Z. H. Sun, Z. K. Liu, J. H. Li, G. A. Tai, S. P. Lau, F. Yan, Infrared Photodetectors Based on CVD-Grown Graphene and $\mathrm{PbS}$ Quantum Dots with Ultrahigh Responsivity. Adv Mater 24, 5878-5883 (2012); published online EpubNov 14 